\title{
Statistics of highly heterogeneous flow fields confined to three-dimensional random porous media
}

\author{
C. Jin, ${ }^{1,2,3, *}$ P. A. Langston,,${ }^{1, \dagger}$ G. E. Pavlovskaya, ${ }^{4, \dagger}$ M. R. Hall, ${ }^{2,3, \S}$ and S. P. Rigby ${ }^{1,2,3, \|}$ \\ ${ }^{1}$ Department of Chemical and Environmental Engineering, Faculty of Engineering, University of Nottingham, NG7 2RD, United Kingdom \\ ${ }^{2}$ GeoEnergy Research Centre (GERC), University of Nottingham, NG7 2RD, United Kingdom \\ ${ }^{3}$ British Geological Survey, Keyworth, Nottingham NG12 5GG, United Kingdom \\ ${ }^{4}$ Sir Peter Mansfield Magnetic Resonance Centre, University of Nottingham, NG7 2RD, United Kingdom \\ (Received 2 September 2015; revised manuscript received 15 November 2015; published 19 January 2016)
}

\begin{abstract}
We present a strong relationship between the microstructural characteristics of, and the fluid velocity fields confined to, three-dimensional random porous materials. The relationship is revealed through simultaneously extracting correlation functions $\mathcal{R}_{u u}(r)$ of the spatial (Eulerian) velocity fields and microstructural two-point correlation functions $\mathcal{S}_{2}(r)$ of the random porous heterogeneous materials. This demonstrates that the effective physical transport properties depend on the characteristics of complex pore structure owing to the relationship between $\mathcal{R}_{u u}(r)$ and $\mathcal{S}_{2}(r)$ revealed in this study. Further, the mean excess plot was used to investigate the right tail of the streamwise velocity component that was found to obey light-tail distributions. Based on the mean excess plot, a generalized Pareto distribution can be used to approximate the positive streamwise velocity distribution.
\end{abstract}

DOI: 10.1103/PhysRevE.93.013122

\section{INTRODUCTION}

The physics of fluids flowing through random porous media is of fundamental importance to a wide range of engineering and scientific fields $[1,2]$. Such diverse fields as enhanced oil recovery (EOR), carbon capture and storage (CCS), contaminant migration in ground-water, and permeation of nutrients in biological tissues require an understanding of, and calculation of, effective physical transport properties resulting from such flow phenomena. As a consequence, the relationships between the effective physical transport properties and the intrinsic complicated pore structure of porous materials have always attracted a considerable amount of attention [2,3]. In the pioneering experimental work of Darcy [4] (known as Darcy's law), the permeability $\kappa$ was determined by the following relationship:

$$
q=-\frac{\kappa}{\mu} \frac{\Delta p}{l}
$$

where $q$ is the flow rate (with the same unit as velocity, $\left.m s^{-1}\right), \mu$ the viscosity of the fluid flowing through the porous medium, with the pressure difference $\Delta p$, along the flow distance of $l$. Obviously, the dependence of permeability on the pore geometry is not accounted for explicitly in Darcy's law. The pore geometry usually consists of an interconnected three-dimensional network of capillary channels of a wide range of sizes and shapes. To address this, a frequently

\footnotetext{
*ezzcj@nottingham.ac.uk

†paul.langston@nottingham.ac.uk

${ }^{\ddagger}$ galina.pavlovskaya@nottingham.ac.uk

${ }^{\S}$ matthew.hall@ nottingham.ac.uk

" sean.rigby@nottingham.ac.uk
}

Published by the American Physical Society under the terms of the Creative Commons Attribution 3.0 License. Further distribution of this work must maintain attribution to the author(s) and the published article's title, journal citation, and DOI. used empirical relationship was proposed by Kozeny [5] and Carman [6,7] with the following general form:

$$
\kappa=\phi \frac{r_{h}^{2}}{k},
$$

where $\phi$ denotes the porosity, $r_{h}$ the hydraulic radius, and $k$ the Kozeny constant associated with the inherent pore geometry. Descriptors of pore geometry typically include a characteristic length scale or correlation functions [8]. There exists a vast amount of work that has attempted to relate the effective physical transport properties, e.g., permeability $\kappa$ and diffusion trapping constant $\gamma$, to the pore geometry using fractals [9], characteristic length scales $[10,11]$, or correlation functions [12-19].

For a single-phase viscous flow, or a single-phase flow with passive scalar tracers [20-22], the effective physical transport properties are determined by the underlying flow field, as they are coupled with the momentum transport equation. However, the underlying flow field is usually determined by the flow conditions. In fluids flowing through porous media in the Darcy regime, the flow field confined to the porous materials are strongly influenced by the complex pore features. This has led to a vast amount of experimental work focusing on measuring the flow field through complex porous media [20,23-27]. Among the previous work, Datta et al. [27] observed that the streamwise velocity component across several packings of beads obeys an exponential distribution. Further, they probed correlation functions of the velocity field. However, they did not characterize the pore space together with the corresponding velocity field.

In this paper, we studied, simultaneously, the correlation function of the flow field and the two-point correlation of the complex pore space. We adopted the mean excess plot (MEP) technique proposed by Ghosh and Resnick [28] and generalized Pareto distributions (GPD) to investigate the right tail of the streamwise velocity component. We used the lattice Boltzmann method (LBM) $[29,30]$ to simulate the viscous pore-scale flows through three different random porous samples. 


\section{PORE-SCALE FLOW SIMULATIONS AND POROUS SAMPLES}

\section{A. Pore-scale flow simulations}

Pore-scale fluid motion within the complex pores of the porous materials is governed by the continuity and NavierStokes equations,

$$
\begin{aligned}
\nabla \cdot \mathbf{u} & =0 \\
\rho\left[\frac{\partial \mathbf{u}}{\partial t}+\mathbf{u} \cdot \nabla \mathbf{u}\right] & =-\nabla p+\mu \nabla^{2} \mathbf{u},
\end{aligned}
$$

where $\rho$ denotes the fluid density, $\mathbf{u}=\left(u_{x}, u_{y}, u_{z}\right)$ the pore velocity vector, $p$ the pressure, and $\mu$ the dynamic viscosity.

We employed the lattice Boltzmann BGK model [31] to simulate the viscous flow within the complex pore spaces. The lattice Boltzmann BGK model is based on the discrete velocity Boltzmann equation

$$
\underbrace{f_{i}\left(\mathbf{x}+\mathbf{e}_{i} \delta t, t+\delta t\right)-f_{i}(\mathbf{x}, t)}_{\text {Streaming }}=\underbrace{-\frac{1}{\tau}\left[f_{i}(\mathbf{x}, t)-f_{i}^{e q}(\mathbf{x}, t)\right]}_{\text {Collision }},
$$

where $\mathbf{x}$ denotes the centroid of the cell, $\tau$ denotes the relaxation time, $f_{i}$ denotes the particle distribution function, which streams along the lattice velocity vector $\mathbf{e}_{i}$; the righthand side of Eq: (5) represents the rate of change of $f_{i}$ resulting from collision between the incoming $f_{i}$ and the equilibrium particle distribution function $f_{i}^{\text {eq }}$. $\delta t$ is assumed to be unity. We used the three-dimensional d3q19 model, so the velocity vector $\mathbf{e}_{i}$ has 19 components. The macroscopic local density $\rho$ and velocity functions $\mathbf{u}$ are evaluated through the moments of the particle distribution functions with respect to $\mathbf{e}_{i}$ :

$$
\begin{aligned}
\rho & =\sum_{i=0} f_{i}, \\
\rho \mathbf{u} & =\sum_{i=0} f_{i} \mathbf{e}_{i} .
\end{aligned}
$$

The relaxation time $\tau$ was chosen to be unity to reduce the viscosity dependence [32] for all the simulations carried out in this study. The pore-scale flow has a Reynolds number $\mathrm{Re} \ll 1$, and was produced by applying a constant small pressure gradient along the flow direction $x$. The no-slip boundary condition was applied at the pore-grain interfaces through the bounce-back technique, and periodic boundary conditions were applied on the $y$ and $z$ directions.

\section{B. Porous samples}

Figure 1 shows computer reconstructions of the three porous materials, chosen to reflect with a wide variation in porosity and pore structures. The corresponding porosity profile for each individual porous sample shown in Fig. 1(d) shows that porosity $\phi$ fluctuates around the averaged porosity over the flow direction $x$ (within \pm 0.05 of the average porosity $\phi$.). Due to different resolutions and length scales associated with the three samples, we present the images and results in pixel or voxel units (it is quite straightforward to convert them to physical units by multiplying the corresponding resolution).
The three porous materials have a cubic lattice of $256^{3}, 512^{3}$, and $512^{3}$, respectively.

The first sample is a realistic random composite porous foam; the second one was built from a simulation of the sedimentation process of bidisperse spherical particles using discrete element modeling (DEM) (see Ref. [33] for details); the third one is a Bentheimer sandstone. The first and third samples were digitized as a parallel stack of two-dimensional images of cross-sections from noninvasive $\mathrm{x}$-ray computed tomography (XCT) measurements; the second sample was digitized through image processing the deposited spherical particles in terms of the centroid and diameter of individual particles. Then the images were segmented into binary images, each pixel of which represents either pore or grain phase. The binary images provide a perfect base for lattice Boltzmann simulation in terms of image-based pore-scale modeling [34].

\section{RESULTS AND DISCUSSION}

\section{A. Determination of the representative elementary volume (REV)}

While microscopically inhomogeneous, the porous samples shown in Fig. 1 exhibit some small regions of prevailing homogeneity. Hence, different regions may exhibit significantly different properties. Therefore, it is crucial to determine that the size of the porous samples under study exceeds the size of the representative element volume with a view to determine the effective transport properties. Using length scales of the representative element volume, which is significantly larger than the inhomogeneities, we can characterize the effective transport properties, e.g., the bulk permeability $\kappa$ (not shown in this study).

Prior work $[35,36]$ has documented different statistical approaches to determine the existence and size of the representative element volume. In this study, a two-point correlation function of the complex pore space was used to characterize the representative element volume.

The three-dimensional random porous materials considered in this study contain two disjointed phases, i.e., pore and grain phases, as shown in Figs. 1(a)-1(c), which can be described through random set models defined in the three-dimensional Euclidean space $\mathbb{R}^{3}$. For a binary cross-section image, $\mathbb{R}^{3}$ is reduced to the two-dimensional Euclidean space $\mathbb{R}^{2}$. The structure of each random porous medium can be regarded as a realization of a time-independent spatial stochastic process or field $(\Omega, \mathcal{F}, \mathbb{P})$ that can be statistically characterized by the indicator function $\mathcal{I}^{i}(\eta)$ [37] for the phase $i \in$ \{pore, grain\}:

$$
\mathcal{I}^{i}(\boldsymbol{\eta})= \begin{cases}1 & \boldsymbol{\eta} \in \mathcal{V}^{i} \\ 0 & \text { otherwise }\end{cases}
$$

where $\mathcal{I}^{i}(\boldsymbol{\eta})$ indicates that any point $\boldsymbol{\eta}$ within the random porous materials takes only values 0 or 1 (a Boolean model) depending upon whether the point $\boldsymbol{\eta}$ belongs to the phase of interest $\mathcal{V}^{i}$ or not. To simplify the notation, we will generally omit the superscript $i$ in $\mathcal{I}^{i}$. To completely characterize the microstructural features of the two-phase porous materials requires knowledge of the canonical $n$-point correlation function $\mathcal{H}_{n}$ [37,38]. However, $\mathcal{H}_{n}$ is computationally intractable in most cases. In this study, we adopted the two-point correlation 
(a)
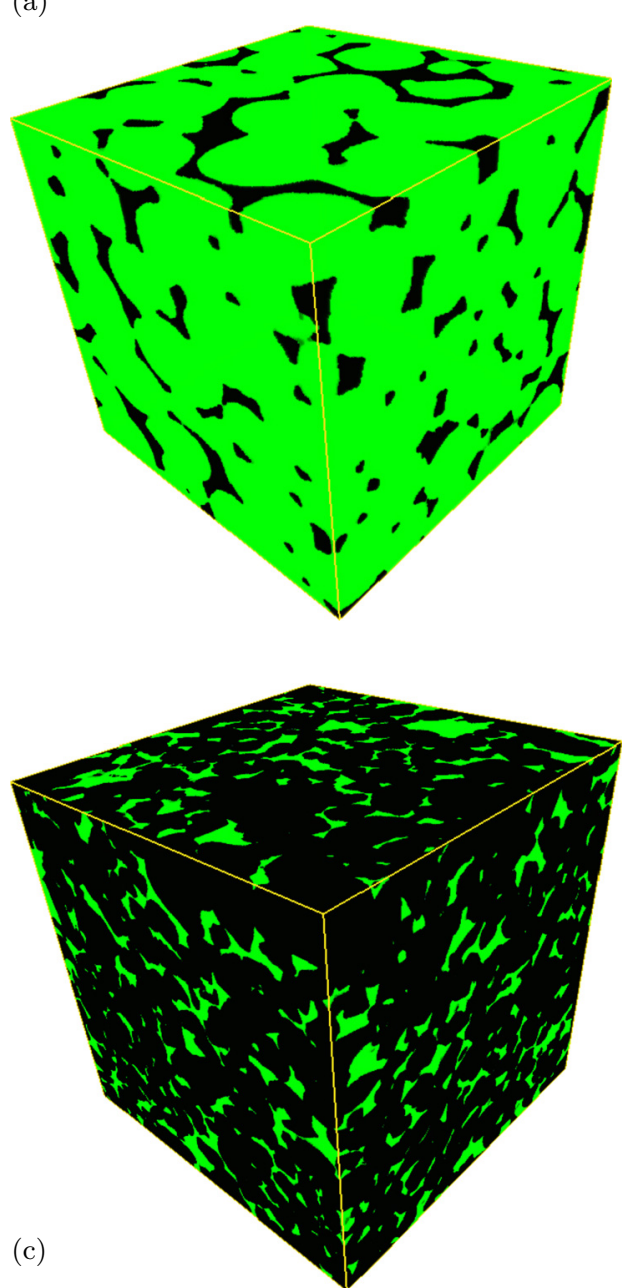

(b)
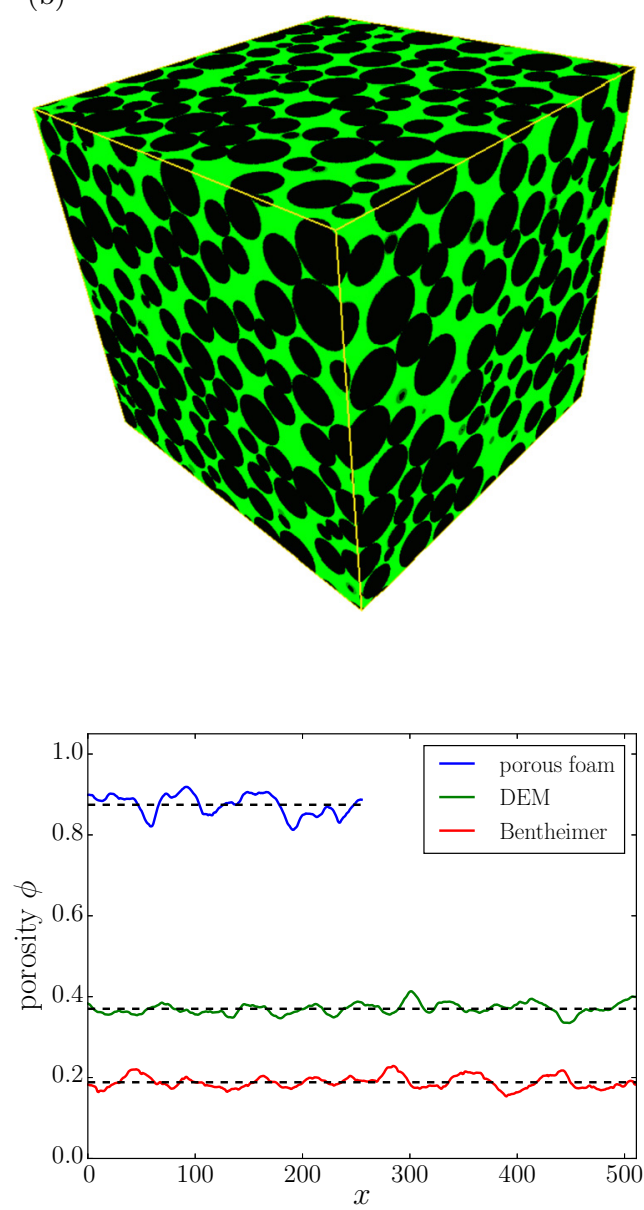

(d)

FIG. 1. 3D binary visualization of random porous samples (a) Porous foam $256^{3}$, (b) Sphere packing generated from DEM $512^{3}$, (c) Bentheimer sandstone $512^{3}$, and (d) plot of porosity as a function of the flow direction $x$ in pixel units. Green indicates pore phase; black, grain phase.

function $\mathcal{S}_{2}(\boldsymbol{\eta}, \boldsymbol{\eta}+\mathbf{r})$ for either of the two phases [39-41], which can be defined as

$$
\mathcal{S}_{2}(\boldsymbol{\eta}, \boldsymbol{\eta}+\mathbf{r})=\langle\mathcal{I}(\boldsymbol{\eta}) \mathcal{I}(\boldsymbol{\eta}+\mathbf{r})\rangle,
$$

where $\langle\cdots\rangle$ denotes an ensemble (volume) average over all positions $\eta$ within the porous material. For an isotropic material, $\mathcal{S}_{2}(\boldsymbol{\eta}, \boldsymbol{\eta}+\mathbf{r})$ is reduced to $\mathcal{S}_{2}(\mathbf{r})$. It is possible to interpret $\mathcal{S}_{2}(\mathbf{r})$ as the probability of finding two points with a separation distance $\|\mathbf{r}\|$ in the same phase (either pore or grain phase). When the separation distance $\|\mathbf{r}\|=0$ in Eq. (9), $\mathcal{S}_{2}$ is reduced to

$$
\lim _{\|\mathbf{r}\| \rightarrow 0} \mathcal{S}_{2}(\mathbf{r})=\phi
$$

which denotes the one-point statistic concerning the porous material, i.e., the porosity $\phi$; when at the opposite extreme $\|\mathbf{r}\| \rightarrow \infty$ in Eq. (9), $\mathcal{S}_{2}$ is reduced to

$$
\lim _{\|\mathbf{r}\| \rightarrow \infty} \mathcal{S}_{2}(\mathbf{r})=\phi^{2} \text {. }
$$

Debye et al. [8] showed that the derivative of $\mathcal{S}_{2}$ at the separation distance zero is equal to $-s / 4$ for three-dimensional isotropic porous media, where $s$ is the specific surface area (interface area per unit volume). For the first three space dimensions, there is a proportionality relation between the the derivative of $\mathcal{S}_{2}$ and the specific surface area as follows:

$$
\mathcal{S}_{2}^{\prime}(0)= \begin{cases}-s / 2, & d=1, \\ -s / \pi, & d=2, \\ -s / 4, & d=3 .\end{cases}
$$

A characteristic pore size $r_{c}$ can be defined based the method proposed by Ref. [14], which is a separation distance where a straight line with the slope equaling $\mathcal{S}_{2}^{\prime}(0)$ intersects the horizontal line $\phi^{2}$ and reads

$$
r_{c}=\frac{\phi(\phi-1)}{\mathcal{S}_{2}^{\prime}(0)} .
$$

We first calculated the one-dimensional $\mathcal{S}_{2}$ of the pore space of all the line sections along the three orthogonal $x, y$, and $z$ directions for the three porous materials $\left(\mathcal{S}_{2}-x, \mathcal{S}_{2}-y\right.$, and $\mathcal{S}_{2}-z$ in $\mathbb{R}^{1}$ ). The results for $\mathcal{S}_{2}-x, \mathcal{S}_{2}-y$, and $\mathcal{S}_{2}-z$ of the pore space were then averaged across all the lines in the $x, y$, and $z$ direction and are shown in Fig. 2. It can be observed that $\mathcal{S}_{2}$ attains the value of $\phi$ at the separation distance $r=0$ and decays rapidly to the asymptotic theoretical value of $\phi^{2}$ in the $x, y$, and $z$ directions for all three porous 
(a)

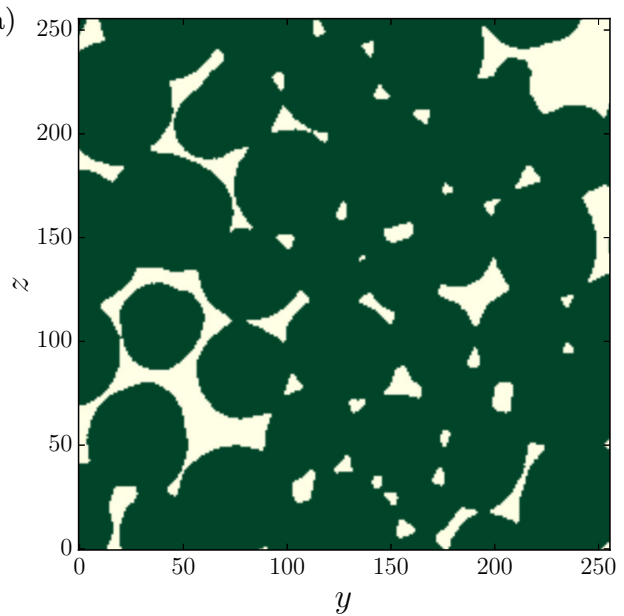

(c)

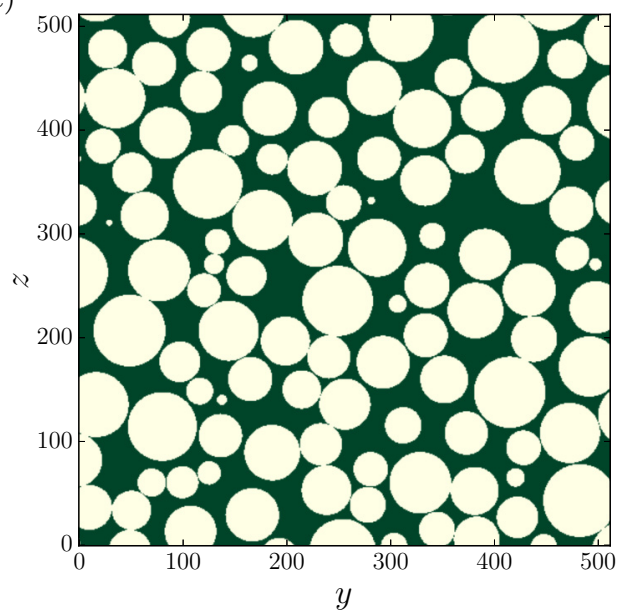

(e)

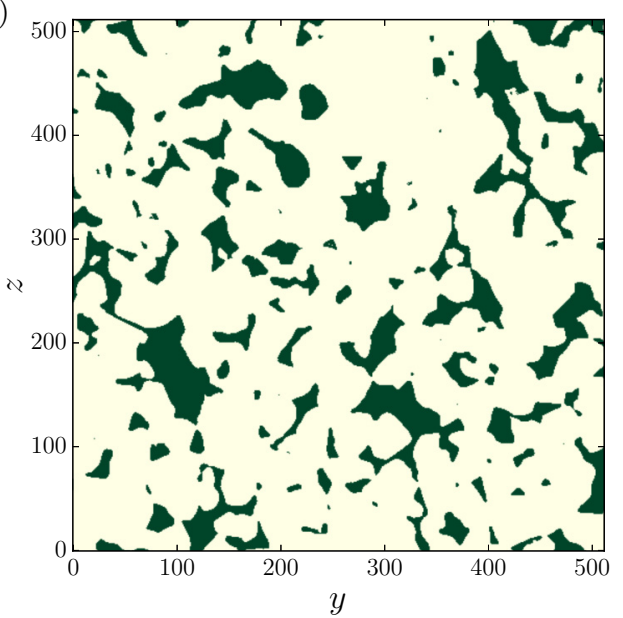

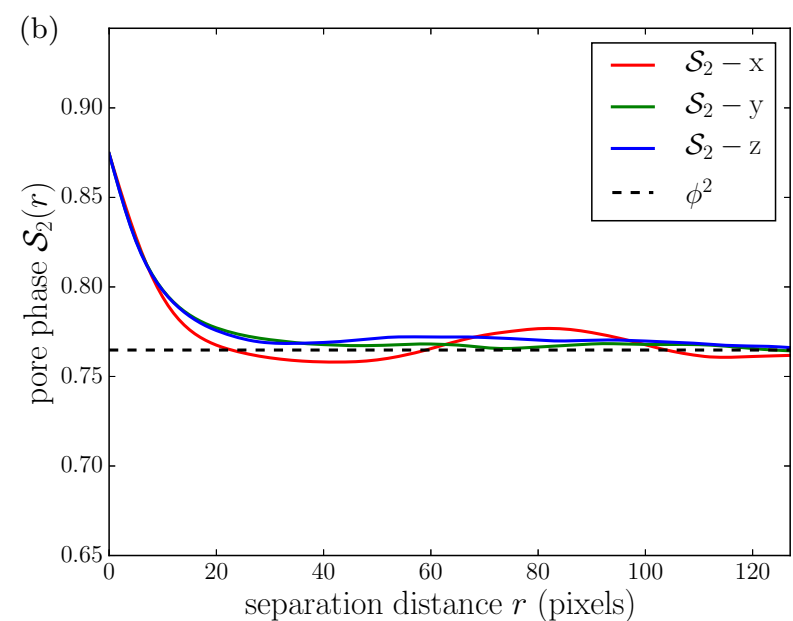

(d)

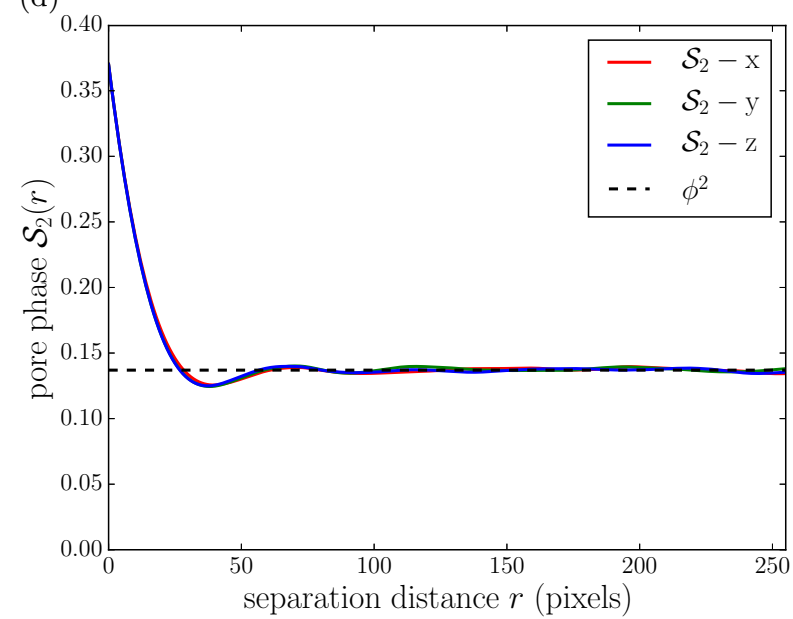

(f)

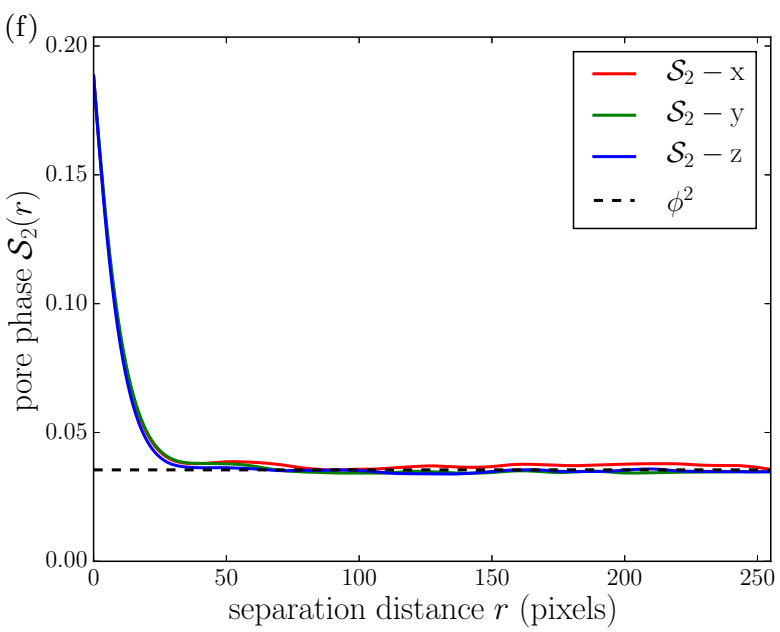

FIG. 2. Binary representation of cross-section of (a) porous foam, (c) sphere packing via DEM, (e) Bentheimer sandstone (green for pore, white for grain); Volume averaged $\mathcal{S}_{2}$ of pore space along orthogonal $x, y$, and $z$ directions for (b) porous foam, (d) sphere packing via DEM, (f) Bentheimer sandstone; separation distance $r$ in pixel units.

materials as expected [42]. The shape of $\mathcal{S}_{2}$ provides an indication of general morphology of the three porous samples. First, $\mathcal{S}_{2}-x, \mathcal{S}_{2}-y$, and $\mathcal{S}_{2}-z$ fall with almost the same rate within a small separation distance, which indicates that $r_{c}$ will be around the same based on $\mathcal{S}_{2}^{\prime}(0)$ in $x, y$, and $z$ direction, respectively. This suggests that the microstructural pores of the three significantly different porous samples are statistically isotropic. Second, $\mathcal{S}_{2}$ curve of the sphere packing via DEM shows a distinct minimum that is a feature of impenetrable sphere packing. This distinct minimum of $\mathcal{S}_{2}(r)$ arises from the excluded-volume effects, which results from the mutual impenetrability of finite-size (hard-core) particles. The separation distance where $\mathcal{S}_{2}(r)$ attains the minimum corresponds roughly with the mean particle size [43]. However, 


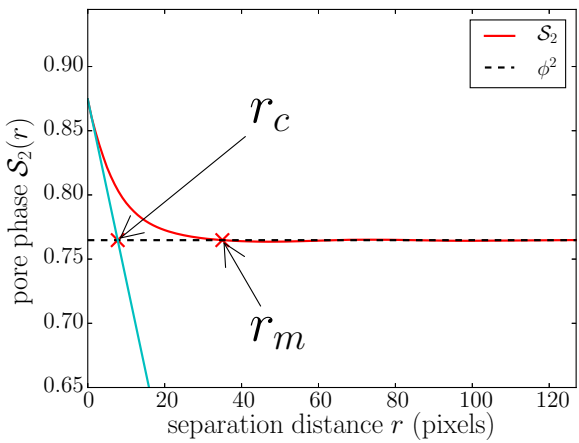

(a)

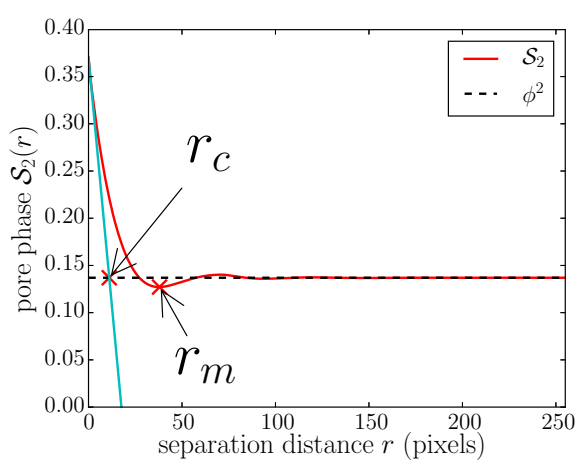

(b)

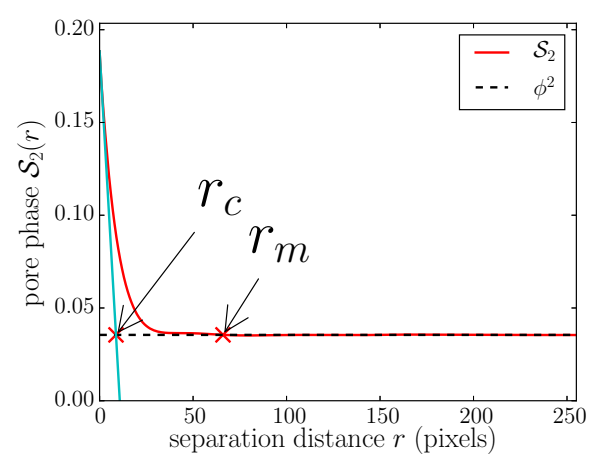

(c)

FIG. 3. Three-dimensional radial averaged $\mathcal{S}_{2}$ of the pore space for (a) porous foam, (b) sphere packing via DEM, and (c) Bentheimer sandstone.

$\mathcal{S}_{2}$ curves of porous foam and Bentheimer sandstone fall monotonically to the asymptotic value $\phi^{2}$, which is a feature of fully penetrable sphere packing.

We also obtained the three-dimensional radial averaged $\mathcal{S}_{2}$ of the pore space for the three samples as shown in Fig. 3. The $\mathcal{S}_{2}$ curves are marked with two characteristic length scales associated with the porous samples. One is the characteristic pore size $r_{c}$, another is the mean grain size $r_{m}$. The porous foam and Bentheimer sandstone can be assumed to be made of polydispersed spheres that interpenetrate mutually to varying degrees. The physical significance of the separation distance where $\mathcal{S}_{2}(r)$ first reaches a local minimum for totally impenetrable particles is that it equals roughly the mean grain size $r_{m}$ as mentioned above. This leads to the definition of an effective mean grain size $r_{m}$ of the porous foam and Bentheimer sandstone.

There are a few features that can be observed from Fig. 3. First, $r_{c}$ is significantly smaller than $r_{m}$ for all three samples (four, three, and seven times smaller, respectively). Further, $\mathcal{S}_{2}$ within the separation distance $r_{c}$ is significantly greater than $\phi^{2}$. It is worth pointing out that $\mathcal{S}_{2}$ is related to the auto-covariance function for the pore phase through the following relationship:

$$
\chi(\mathbf{r})=\langle[\mathcal{I}(\boldsymbol{\eta})-\phi][\mathcal{I}(\boldsymbol{\eta}+\boldsymbol{r})-\phi]\rangle=\mathcal{S}_{2}(\boldsymbol{r})-\phi^{2} .
$$

Therefore, points within pore phase separated by a distance that is smaller than $r_{c}$ are positively correlated as $\mathcal{S}_{2}$ is of having values falling within the interval of $\left(\phi^{2}, \phi\right]$ (in light of $\left.\chi(\mathbf{r}) \in\left(0, \phi-\phi^{2}\right]\right)$. On the other hand, points within pore phase separated by a distance that is much larger than $r_{m}$ are uncorrelated as $\mathcal{S}_{2}$ is of having values very close to $\phi^{2}$. Second, as shown in Fig. 3, the size of the three porous materials is significantly greater than $r_{c}$ (given that the plot length is the half of the system size due to periodic boundary conditions). This ensures sufficiently good statistics for the transport properties as the volume of each sample can be regarded as a representative elementary volume [44]. The estimates for $s, r_{c}$, and $r_{m}$ are shown in the Table I. The porosity of the porous foam is of the highest of the three samples; however, this does not necessarily mean it attains the largest $r_{c}$ as $r_{c}$ depends on both $\mathcal{S}_{2}^{\prime}(0)$ and $\phi$.

\section{B. Correlation functions of the pore spaces and velocity fields}

We obtained the flow fields confined to the complex pore spaces of the three samples via LB simulations with no-slip boundary conditions at the pore-grain interface. The flow was driven by a constant pressure gradient along the streamwise $x$ direction. Figure 4 shows representative fluid particles flowing through the heterogeneous pore spaces. It can be observed that the trajectories of fluid particles are tortuous and preferential. The velocity magnitude also exhibits significant variations that arise due to the confined and heterogeneous pore space.

Figure 5 shows streamwise velocity contours, $\mathcal{S}_{2}$ of the pore space along with the corresponding Eulerian correlation functions of the velocity field for the three porous materials. The velocity contours shown in Figs. 5(a), 5(c), and 5(e) are based on the binary representation of the cross-section shown in Figs. 5(b), 5(d), and 5(f). $\mathcal{S}_{2}$ shown in Figs. 5(a), 5(c), and $5(\mathrm{e})$ were calculated for the corresponding pore phase in $\mathbb{R}^{2}$. The Eulerian correlation function of the interstitial streamwise velocity field can be calculated in terms of the following expression:

$$
\mathcal{R}_{u u}(\boldsymbol{\eta}, \boldsymbol{\eta}+\boldsymbol{r})=\frac{\langle[(u(\boldsymbol{\eta})-\bar{u})(u(\boldsymbol{\eta}+\boldsymbol{r})-\bar{u})]\rangle}{\sigma_{u}^{2}},
$$

where $\bar{u}$ and $\sigma_{u}^{2}$ denote the mean value and variance of the interstitial streamwise velocity for the whole cross-section, respectively. Comparing the velocity contours to the original binary representation of the corresponding cross-sections, we can observe that there are no isolated pores (pore surrounded by the grain phase completely), as all the pores are filled with fluids. The velocities within each individual pore for all three porous materials show significant variations across the plane, even for the Bentheimer sandstone with the lowest porosity. Very sporadic, or localized high velocity, is observed in a few random pores, which looks similar to "burst" of turbulence or may relate to the pore-scale intermittent velocity structure $[45,46]$. These high fluid velocity regions stretch across several pore length scales and look very similar to the large-scale spatially correlated "coherent structure" of turbulence [47], though the flow under study is a laminar one overall and

TABLE I. Estimates for $s, r_{c}$, and $r_{m}$.

\begin{tabular}{lccc}
\hline \hline Samples & $s{\text { [pixel }]^{-1}}$ & $r_{c}$ [pixel $]$ & $r_{m}$ [pixel] \\
\hline Porous foam & 0.00352 & 7.87 & 35.1 \\
Sphere packing & 0.00525 & 11.1 & 38.2 \\
Bentheimer sandstone & 0.00436 & 8.77 & 66.2 \\
\hline \hline
\end{tabular}




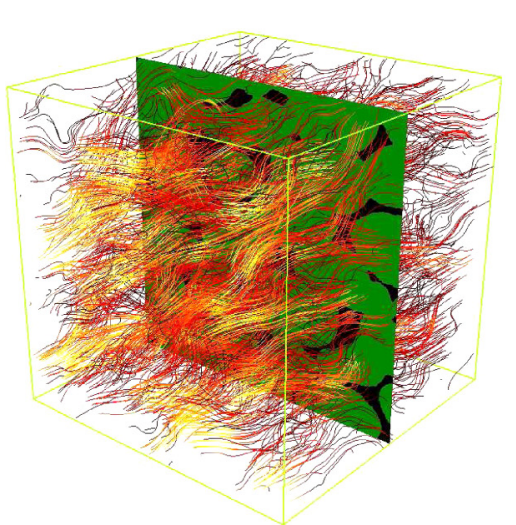

(a)

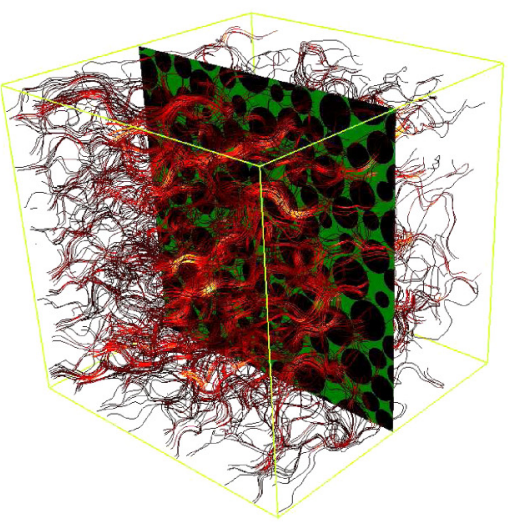

(b)

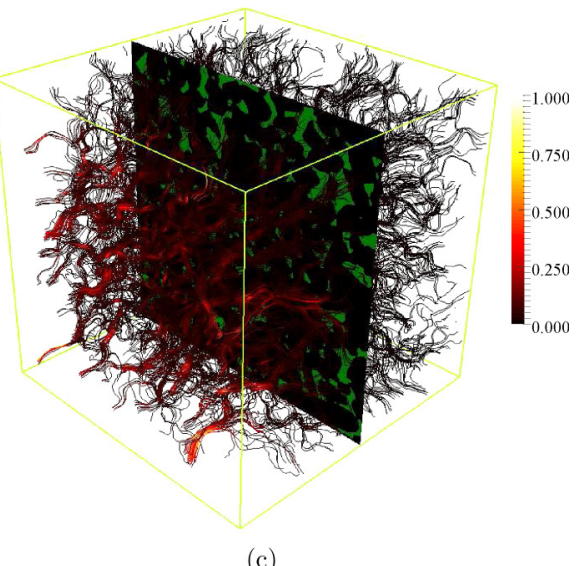

(c)

FIG. 4. Representative fluid particles through the pore space for (a) porous foam, (b) sphere packing via DEM, and (c) Bentheimer sandstone, visualized in terms of normalized velocity magnitude.

for individual pore spaces. Moreover, negative velocities are observed in flows through the three porous materials (indicated in the inset images), which arises due to recirculating resulting from centrifugal action as fluid particles try to navigate through the tortuous pore space with a wide range of sizes and shapes (second flow resulting from centrifugal action). The percentage of fluid voxels with negative interstitial streamwise velocity was also quantified and shown in the Table II. The Bentheimer sandstone has the highest percentage of negative streamwise velocity, which arises due to the most complex underlying pore space. Negative streamwise velocities have also been reported for flows through porous media in Refs. [27,48,49]. The stagnation zones within the second flow region may be related to non-Fickian transport [21] or the phenomena of hold-up dispersion [20,50].

Further, we computed $\mathcal{R}_{u u}(r)$ based on Eq. (15) to characterize the spatial (Eulerian) correlation of the velocity fields within the three porous materials as shown in Fig. 5. Unlike Datta et al. [27], who studied correlation functions of the velocity field along the flow direction and the Lagrangian correlation functions of velocities investigated by Le Borgne et al., the velocity field used for computing $\mathcal{R}_{u u}(r)$ lies in a cross-section perpendicular to the flow direction. This is more appropriate for investigating the relationship between the velocity field confined to pore space and the pore space itself, as it may reduce the spurious correlation resulting from the same fluid stream line in $\mathcal{R}_{u u}(r)$. As discussed before, $\mathcal{S}_{2}$ is an important quantitative measure of spatial correlation of the pore space, and its properties are crucial to understand the complex pore geometry. Now with the help of $\mathcal{R}_{u u}(r)$, which relates a characteristic length scale of velocity to the random flow field, we try to relate the characteristic length scale of the flow field to the inherent length scale of the

TABLE II. Percentage of negative streamwise velocity.

\begin{tabular}{lc}
\hline \hline Samples & Percentage \\
\hline Porous foam & $1.25 \%$ \\
Sphere packing & $11.2 \%$ \\
Bentheimer sandstone & $12.8 \%$ \\
\hline \hline
\end{tabular}

pore space itself embedded in $\mathcal{S}_{2}$. $\mathcal{S}_{2}$ for the cross-sections shown in Figs. 5(a), 5(c), and 5(e) exhibit rather different characteristics. For example, for the pore space within the sphere packing resulting from DEM, $\mathcal{S}_{2}$ exhibits quite obvious oscillations (distinct minimum) with a very similar periodicity, since the distinct peaks of the $\mathcal{S}_{2}$ and $\mathcal{R}_{u u}(r)$ appear nearly at the identical positions. This indicates spatial correlations between the pore space resulting from the excluded-volume effects of the nonoverlapping spheres [37]. In the case of the porous foam, $\mathcal{S}_{2}$ exhibits relatively weaker oscillations, which indicates that part of the spherical pores mix with others. However, in the case of Bentheimer sandstone, $\mathcal{S}_{2}$ decays to the asymptotic theoretical value $\phi^{2}$ with very small fluctuations, which indicates that the length scale of random pores are quite short ranged. However, irregular and heterogeneous clusters can be observed in both the pore and grain phase for the Bentheimer sandstone. Finally, $\mathcal{R}(r)$ is greater than zero for all three samples, which indicates that the Eulerian velocity field is always positively correlated.

Comparing $\mathcal{R}_{u и}(r)$ with $\mathcal{S}_{2}$ for each individual case, we can observe that the Eulerian correlation functions $\mathcal{R}_{u u}(r)$ and $\mathcal{S}_{2}$ for the three samples are all short ranged and decay exponentially. Further, it can be observed that the distinct oscillations of $\mathcal{R}_{u u}(r)$ follow a similar pattern to those of $\mathcal{S}_{2}$, in particular for the case of sphere packing. This interesting feature we want to emphasize indicates that the spatially randomly varying flow field confined to the pore space is remarkably related to the spatial correlation of the pore space. It is consistent with the report by Lester et al. [51], that chaotic flow dynamics within porous media, even under steady-flow conditions, result from the intrinsic topological complexity of the porous media.

\section{Mean excess plot of, and generalized Pareto distribution approximation for, streamwise velocities}

Probability density functions (p.d.f.) of the streamwise velocity component reported in previous studies [27,48,49] indicated that it is of positive skewness and exponential decay. In this study, we employed the mean excess plot [28] to further study the probability density functions of the positive streamwise velocity component (right tail). The mean excess 
(a)

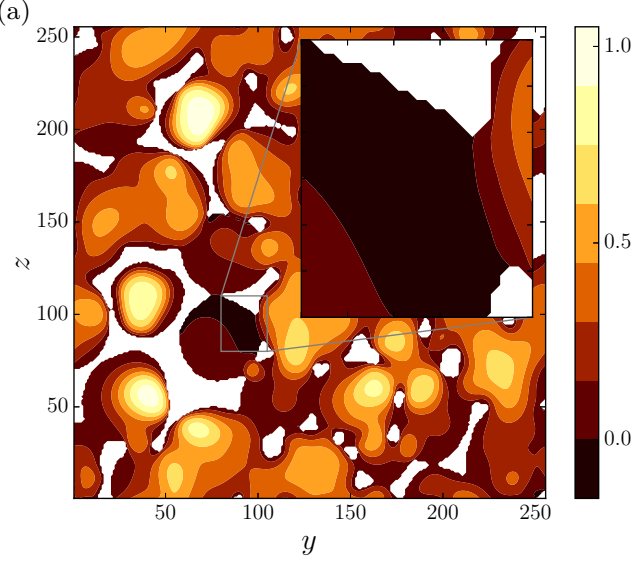

(c)

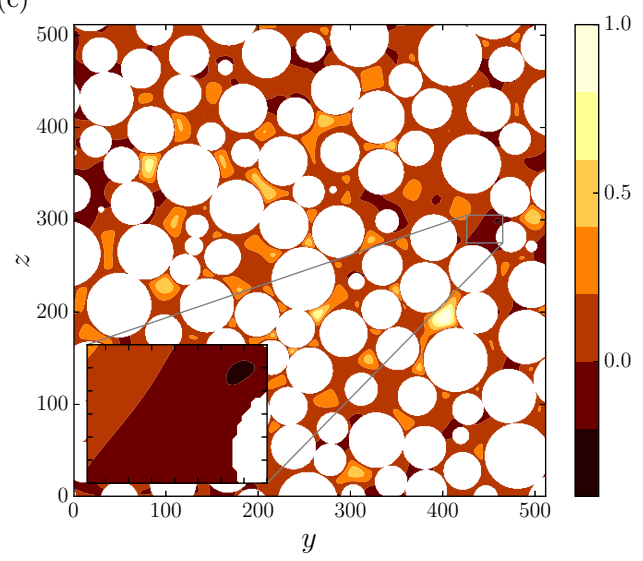

(e)

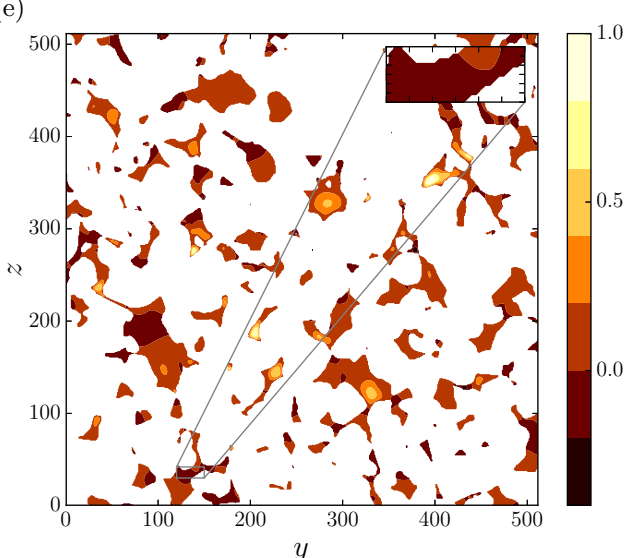

(b)

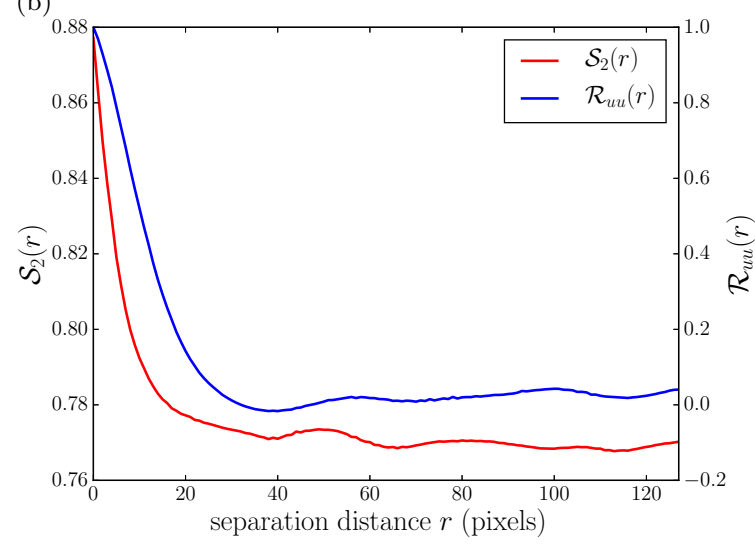

(d)

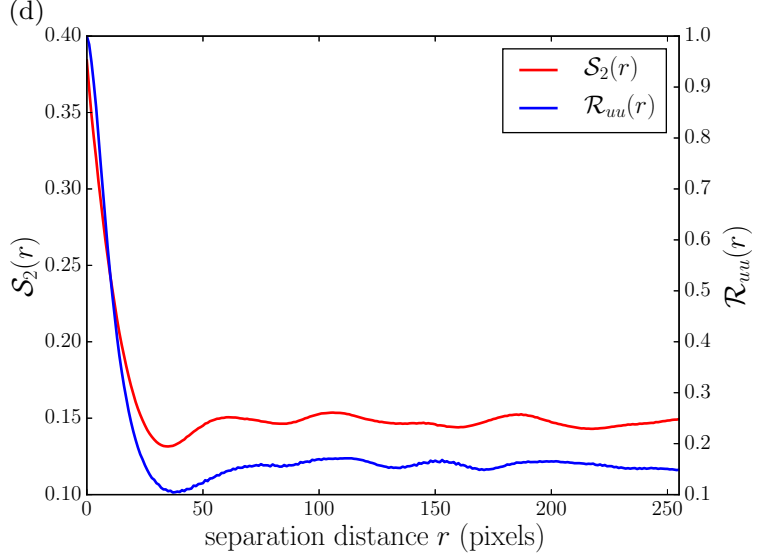

(f)

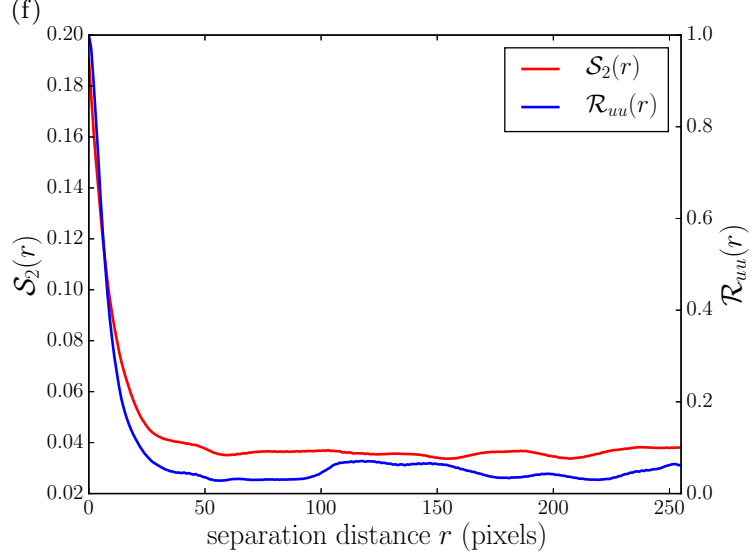

FIG. 5. Contour of streamwise velocity of (a) porous foam, (c) sphere packing via DEM, and (e) Bentheimer sandstone (grain phase marked out using white color); $\mathcal{S}_{2}$ along $\mathcal{R}_{u и}$ of (b) porous foam, (d) sphere packing via DEM, and (f) Bentheimer sandstone; separation distance $r$ in pixel units.

plot is defined in terms of the behavior of the mean excess function as follows:

$$
\mathcal{M}(u)=\mathbb{E}[\Xi-u \mid \Xi>u]=\frac{\int_{u}^{\theta_{F}} F(\theta) d \theta}{\bar{F}(u)},
$$

where $\Xi$ denotes a random variable conditioning on the threshold value $u$ ( $u$ is adopted as we study the velocity component) with distribution function $F(\theta)$, tail distribution function $\bar{F}(\theta)=1-F(\theta)$, and right end point $\theta_{F}$. As discussed by Ghosh and Resnick [28], the mean excess plot can be used to distinguish heavy and light tail distributions of random variables in terms of its trend. For example, a heavy tail distribution, e.g., the lognormal distribution or power law, typically tends to infinity; an exponential distribution $\operatorname{Exp}(\lambda)$ with $\mathcal{M}(u)=\lambda^{-1}$, a horizontal line for the whole variable range; a light tail distribution tends to zero.

Figure 6 shows that the probability density distributions (p.d.f.) and mean excess plots of the positive streamwise 
(a)

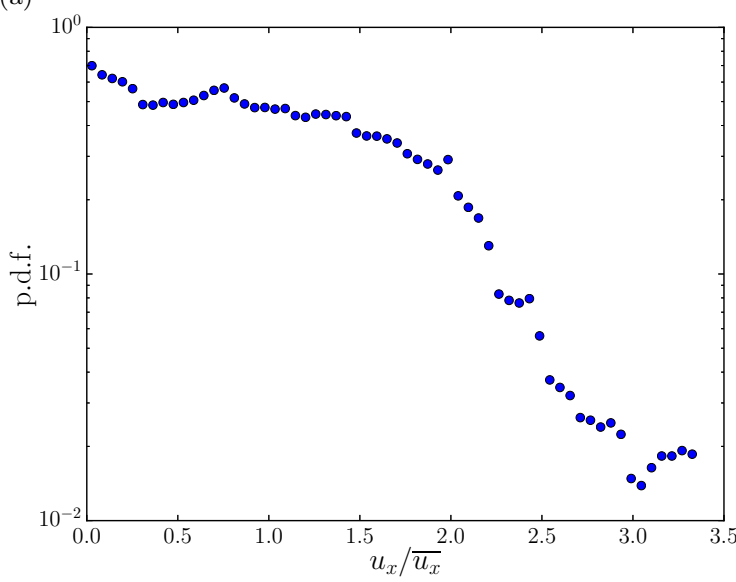

(c)

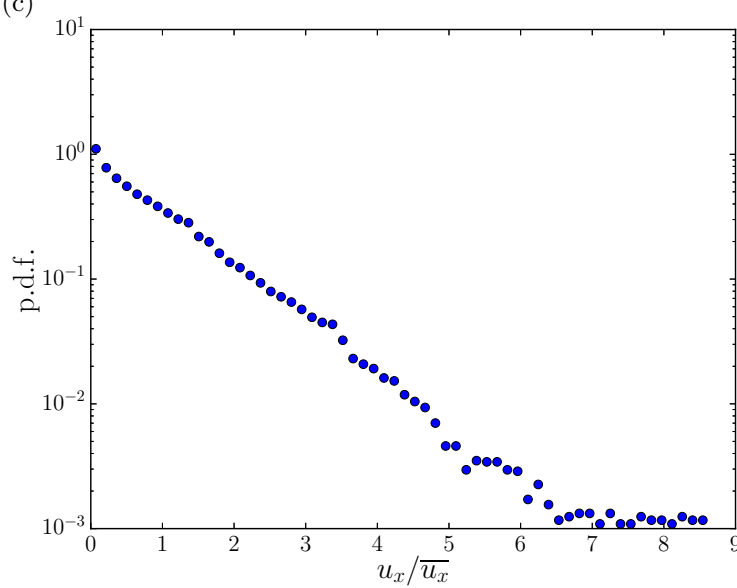

(e)

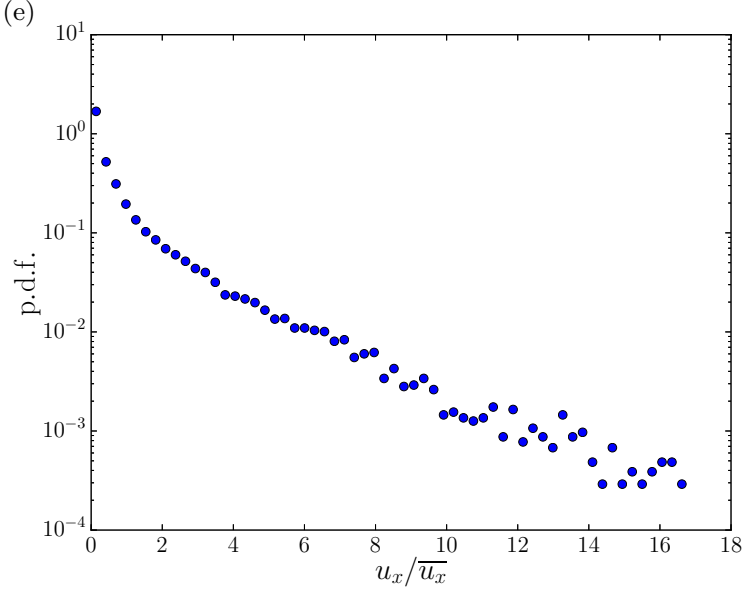

(b)

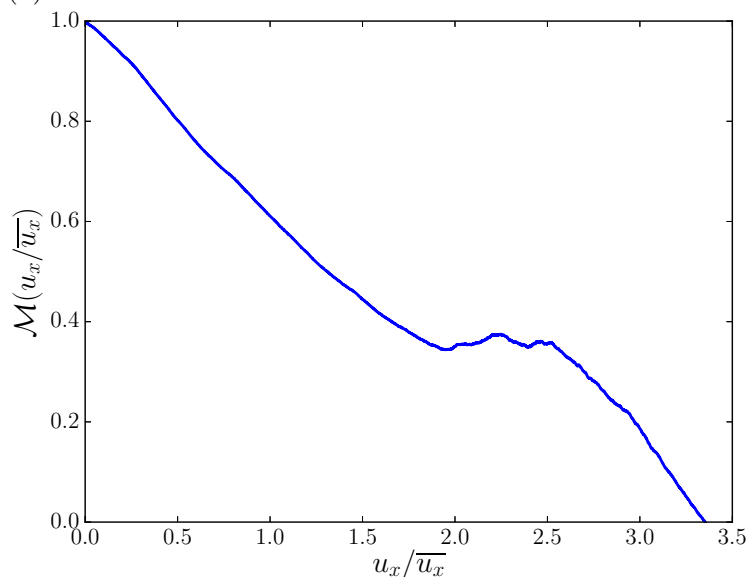

(d)

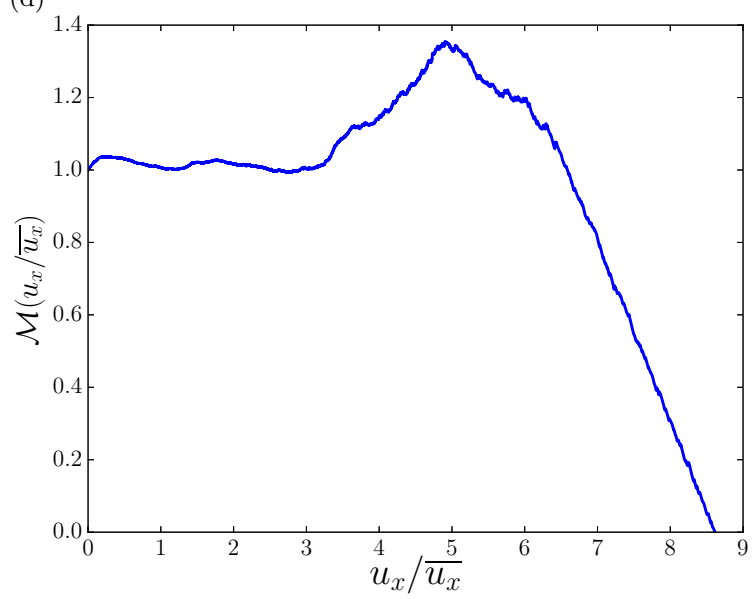

(f)

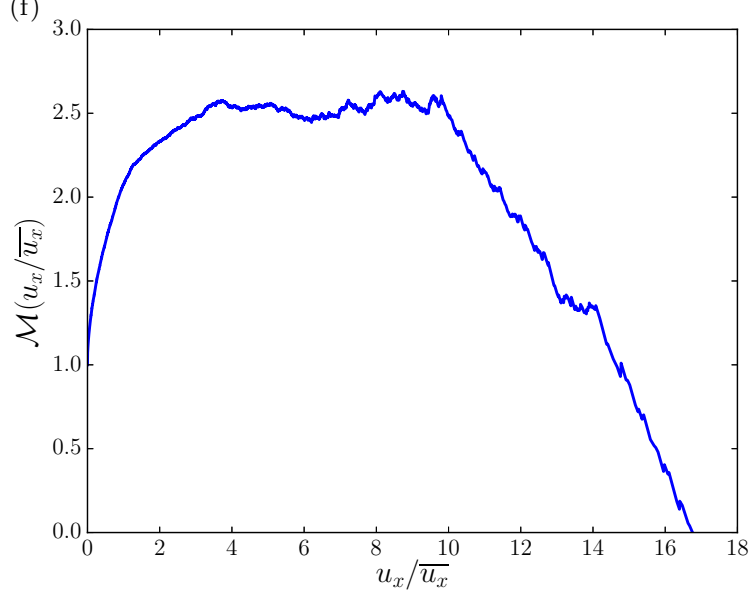

FIG. 6. Probability density functions (p.d.f.) and mean excess plots of positive streamwise velocity of (a, b) porous foam, (c, d) sphere packing via DEM, and (e, f) Bentheimer sandstone.

velocity component (right tail) normalized through the averaged value. As can be observed from the p.d.f. plots shown in Figs. 6(a), 6(c), and 6(e), the streamwise velocity is widely distributed. Further, the lower the porosity, the wider the distribution. This can be explained by the fact that there exist more slow flow regions within the more complex pore space. This result is also consistent with the wide distribution of experimental measurements shown by Datta et al. [27] and simulation results shown by Bijeljic et al. [52] and Siena et al. [49]. As can be seen through the mean excess plots shown in Figs. 6(b), 6(d), and 6(f), the positive streamwise velocity components for the three porous materials show very interesting features. First, the curves of the mean excess plots attain unity at the threshold value of 0 as the streamwise velocities were normalized by the average value. Second, the curves of the mean excess plots tend to zero at the right end point of the streamwise velocity. This indicates that the streamwise velocity components obey light tail distributions 
[28]. Second, each figure shows a region of relatively constant slope, which indicates that the velocity within this region can be approximated by an exponential distribution. This is consistent with the exponential approximation for the positive streamwise velocity studied by Siena et al. [49]. However, each mean excess function shows strikingly different slopes for the whole right tail. Particularly, opposite signs can be observed on the slopes of the mean excess function of streamwise velocity of flow through the sphere packing and Bentheimer sandstone. Third, the mean excess function is close to linear for high values of the threshold $u$. This justifies that using a generalized Pareto distribution is appropriate to describe the statistical properties of right tail of the streamwise velocity.

A random variable $\Xi$ has a generalized Pareto distribution (GPD) if it has a cumulative distribution function (c.d.f.) of the form

$$
\mathcal{G}_{\xi, \beta}(\theta)= \begin{cases}1-(1+\xi \theta / \beta)^{-1 / \xi} & \text { if } \xi \neq 0, \\ 1-\exp (-\theta / \beta) & \text { if } \xi=0,\end{cases}
$$

where $\xi$ denotes the shape parameter of the distribution and $\beta$ the scale parameter $(\beta>0)$. The range of $\theta$ is $\theta>0$ for $\xi \geqslant 0$ and $0<\theta<-\beta / \xi$ for $\xi<0$. Substituting the generalized Pareto distribution (GPD) $\mathcal{G}_{\xi, \beta}(\theta)$ for the distribution function $F(\theta)$, from the mean excess function Eq. (16), we have

$$
\begin{aligned}
\mathbb{P} & (\Xi-u<\theta \mid \Xi>u) \\
& =\frac{\mathbb{P}(u<\Xi \leqslant \theta+u)}{\mathbb{P}(\Xi>u)} \\
& =\frac{\mathcal{G}_{\xi, \beta}(\theta+u)-\mathcal{G}_{\xi, \beta}(u)}{1-\mathcal{G}_{\xi, \beta}(u)} \\
& =\frac{(1+\xi u / \beta)^{-1 / \xi}-(1+\xi(\theta+u) / \beta)^{-1 / \xi}}{(1+\xi u / \beta)^{-1 / \xi}} \\
& =1-(1+\xi \theta /(\beta+\xi u))^{-1 / \xi},
\end{aligned}
$$

which indicates that if $\theta$ is a generalized Pareto distribution of $\mathcal{G}_{\xi, \beta}$, then $\theta-u$, conditioning on $\theta>u$, is a generalized Pareto distribution of $\mathcal{G}_{\xi, \beta+\xi u}$. This further confirms that if a generalized Pareto distribution is consistent with a set of data for a given threshold value, it then must be consistent with the data for all values greater than the given threshold value.

The method of moments proposed by Hosking and Wallis [53] was used to estimate the parameter $\xi$ and $\beta$. The values of
TABLE III. Values of parameter $\xi$ and $\beta$.

\begin{tabular}{lcc}
\hline \hline Samples & $\xi$ & $\beta$ \\
\hline Porous foam & -0.6046 & 1.567 \\
Sphere packing & -0.00955 & 1.044 \\
Bentheimer sandstone & 0.2003 & 1.345 \\
\hline \hline
\end{tabular}

$\xi$ and $\beta$ for the three samples are shown in Table III. Figure 7 shows the generalized Pareto distribution approximation of positive streamwise velocities. It can be observed that the approximation is in good agreement with the simulated velocity data that display a near exponential behavior. We also noted that a stretched exponential distribution was used to fit the right tail of the streamwise velocity component by Siena et al. [49]. Siena et al. also found that there is a strong correlation between the key parameters controlling the decay of probability distribution functions of the streamwise velocity component and pore sizes. It should be possible, therefore, to investigate the relationship of the generalized Pareto distribution for the streamwise velocity component and the probability density function of pore sizes for the three random porous samples presented in this study.

\section{SUMMARY AND REMARKS}

In this paper, we used the lattice Boltzmann method to simulate pore-scale flows within three porous samples. The three porous samples were determined as statistically isotropic and each has size greater than the size of the corresponding representative element volume as a result of the two-point correlation function of the complex pore space.

Through investigating the correlation functions of the complex pore spaces and velocity fields of three porous materials, we found that the spatially varying random velocity field is strongly influenced by the inherent pore structures, especially the length scale for the velocity field is directly related to the length scale of the underlying complex pore space. This is crucial to understand transport phenomena within porous media. We may define different corresponding characteristic length scales in terms of $\mathcal{R}_{u u}(r)$, e.g., the convective length scale $L_{c}$ and the diffusive length scale $L_{d}$. However, we plan to calculate the permeability $\kappa$ and explore the relationship between the length scales of flow field or the pore space

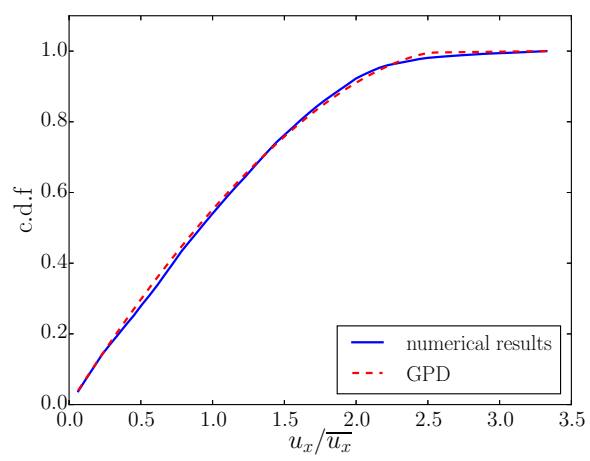

(a)

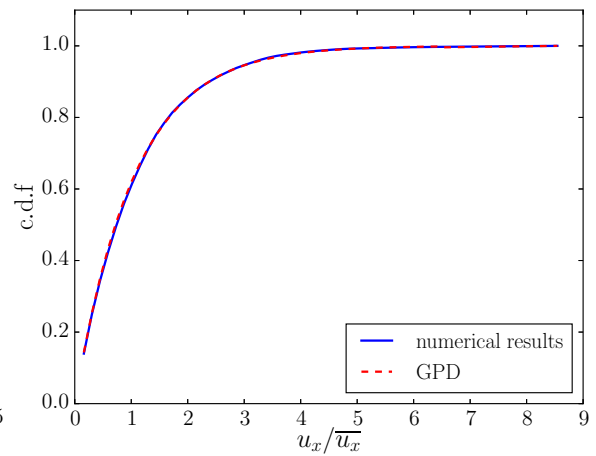

(b)

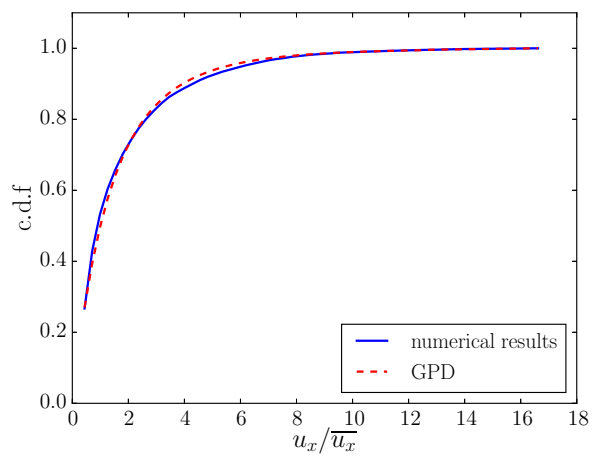

(c)

FIG. 7. Cumulative distribution functions (c.d.f.) and generalized Pareto distribution approximation for positive streamwise velocity of (a) porous foam, (b) sphere packing via DEM, and (c) Bentheimer sandstone. 
and permeability. Future work should therefore be focusing on evaluating whether there might be another prediction of permeability for a wide range of porous media.

Through the mean excess plots of the positive streamwise velocity components, we confirmed that they obey light-tail distributions and can be approximated by a generalized Pareto distribution.

\section{ACKNOWLEDGMENTS}

This work was supported by the Engineering and Physical Sciences Research Council (Grant No. EP/J008753/1). We thank Dr. Martin Corfield for assisting obtaining the XCT images of the porous samples. The computational resources were provided by the Minerva HPC computational nodes of the University of Nottingham.
[1] M. Sahimi, Flow and Transport in Porous Media and Fractured Rock: From Classical Methods to Modern Approaches (Wiley VCH, Berlin, 1995).

[2] F. A. Dullien, Porous Media: Fluid Transport and Pore Structure (Academic Press, New York, 1991).

[3] P. Adler, Porous Media: Geometry and Transports (ButterworthHeinemann, Stoneham, MA, 1992).

[4] H. Darcy, Les fontaines publiques de la ville de Dijon: Exposition et application (Victor Dalmont, Paris, 1856).

[5] J. Kozeny, Sitzungsber. Akad. Wiss. Wien. 136, 271 (1927).

[6] P. Carman, J. Agricult. Sci. 29, 262 (1937).

[7] P. Carman, Flow of Gases Through Porous Media (Butterworths Scientific Publications, London, 1956).

[8] P. Debye, H. Anderson Jr, and H. Brumberger, J. Appl. Phys. 28, 679 (1957).

[9] A. Thompson, A. Katz, and C. Krohn, Adv. Phys. 36, 625 (1987).

[10] C. Scholz, F. Wirner, J. Götz, U. Rüde, G. E. Schröder-Turk, K. Mecke, and C. Bechinger, Phys. Rev. Lett. 109, 264504 (2012).

[11] C. Scholz, F. Wirner, M. A. Klatt, D. Hirneise, G. E. SchröderTurk, K. Mecke, and C. Bechinger, Phys. Rev. E 92, 043023 (2015).

[12] S. Prager, Phys. Fluids 4, 1477 (1961).

[13] J. G. Berryman, J. Chem. Phys. 82, 1459 (1985).

[14] J. G. Berryman and S. C. Blair, J. Appl. Phys. 60, 1930 (1986).

[15] J. Rubinstein and S. Torquato, J. Fluid Mech. 206, 25 (1989).

[16] S. Torquato, Appl. Mech. Rev. 44, 37 (1991).

[17] P. P. Mitra, P. N. Sen, L. M. Schwartz, and P. Le Doussal, Phys. Rev. Lett. 68, 3555 (1992).

[18] S. C. Blair, P. A. Berge, and J. G. Berryman, J. Geophys. Res.: Solid Earth 101, 20359 (1996).

[19] S. Torquato and D. C. Pham, Phys. Rev. Lett. 92, 255505 (2004).

[20] D. Kandhai, D. Hlushkou, A. G. Hoekstra, P. M. A. Sloot, H. Van As, and U. Tallarek, Phys. Rev. Lett. 88, 234501 (2002).

[21] B. Bijeljic, P. Mostaghimi, and M. J. Blunt, Phys. Rev. Lett. 107, 204502 (2011).

[22] T. Le Borgne, M. Dentz, and J. Carrera, Phys. Rev. Lett. 101, 090601 (2008).

[23] S. Saleh, J. Thovert, and P. Adler, Exp. Fluids 12, 210 (1992).

[24] Y. Kutsovsky, L. Scriven, H. Davis, and B. Hammer, Phys. Fluids 8, 863 (1996).

[25] J. D. Seymour and P. T. Callaghan, AIChE J. 43, 2096 (1997).

[26] B. Manz, L. Gladden, and P. Warren, AIChE J. 45, 1845 (1999).

[27] S. S. Datta, H. Chiang, T. S. Ramakrishnan, and D. A. Weitz, Phys. Rev. Lett. 111, 064501 (2013).

[28] S. Ghosh and S. Resnick, Stoch. Process. Appl. 120, 1492 (2010).
[29] D. H. Rothman, Geophysics 53, 509 (1988).

[30] S. Chen and G. D. Doolen, Annu. Rev. Fluid Mech. 30, 329 (1998).

[31] S. Succi, The Lattice Boltzmann Equation for Fluid Dynamics and Beyond (Oxford University Press, Oxford, 2001).

[32] C. Pan, L.-S. Luo, and C. T. Miller, Comput. Fluids 35, 898 (2006).

[33] P. Langston, U. Tüzün, and D. Heyes, Chem. Eng. Sci. 50, 967 (1995).

[34] M. J. Blunt, B. Bijeljic, H. Dong, O. Gharbi, S. Iglauer, P. Mostaghimi, A. Paluszny, and C. Pentland, Adv. Water Res. 51, 197 (2013).

[35] I. Taina, R. Heck, and T. Elliot, Can. J. Soil Sci. 88, 1 (2008).

[36] O. Rozenbaum and S. R. du Roscoat, Phys. Rev. E 89, 053304 (2014).

[37] S. Torquato, Random Heterogeneous Materials: Microstructure and Macroscopic Properties, Vol. 16 (Springer Science \& Business Media, Berlin, 2002).

[38] S. Torquato and G. Stell, J. Chem. Phys. 77, 2071 (1982).

[39] Y. Jiao, F. H. Stillinger, and S. Torquato, Phys. Rev. E 76, 031110 (2007).

[40] Y. Jiao, F. H. Stillinger, and S. Torquato, Phys. Rev. E 77, 031135 (2008).

[41] Y. Jiao, F. H. Stillinger, and S. Torquato, Proc. Natl. Acad. Sci. USA 106, 17634 (2009).

[42] J. G. Berryman, J. Appl. Phys. 57, 2374 (1985).

[43] S. Torquato and G. Stell, J. Chem. Phys. 82, 980 (1985).

[44] J. Bear, Dynamics of Fluids in Porous Media (Dover Civil and Mechanical Engineering, London, 1989).

[45] P. de Anna, T. Le Borgne, M. Dentz, A. M. Tartakovsky, D. Bolster, and P. Davy, Phys. Rev. Lett. 110, 184502 (2013).

[46] P. K. Kang, P. Anna, J. P. Nunes, B. Bijeljic, M. J. Blunt, and R. Juanes, Geophys. Res. Lett. 41, 6184 (2014).

[47] H. Tennekes and J. L. Lumley, A First Course in Turbulence (MIT Press, Cambridge, MA, 1972).

[48] R. S. Maier, D. Kroll, Y. Kutsovsky, H. Davis, and R. S. Bernard, Phys. Fluids 10, 60 (1998).

[49] M. Siena, M. Riva, J. D. Hyman, C. L. Winter, and A. Guadagnini, Phys. Rev. E 89, 013018 (2014).

[50] S. Whitaker, AIChE J. 13, 420 (1967).

[51] D. R. Lester, G. Metcalfe, and M. G. Trefry, Phys. Rev. Lett. 111, 174101 (2013).

[52] B. Bijeljic, A. Raeini, P. Mostaghimi, and M. J. Blunt, Phys. Rev. E 87, 013011 (2013).

[53] J. R. Hosking and J. R. Wallis, Technometrics 29, 339 (1987). 